\section{Schistosomiasis and Presinusoidal Portal Hypertension}

M. Willems ${ }^{1}, H$. van Buuren ${ }^{1}$, P. Zondervan ${ }^{2}$, S. Gratema ${ }^{2}$

${ }^{1}$ Dept. of Hepatogastroenterology, University Hospital Rotterdam, The Netherlands

2 Dept. of Pathology,

University Hospital Rotterdam, The Netherlands

\section{Corresponding Author}

M. Willems, M.D., Ph.D.

Dept. of Hepatogastroenterology Erasmus University Hospital Rotterdam PO Box 2040

3000 CA Rotterdam

The Netherlands

Fax: + 31-10-4365916

E-mail: willems@mdl.azr.nl

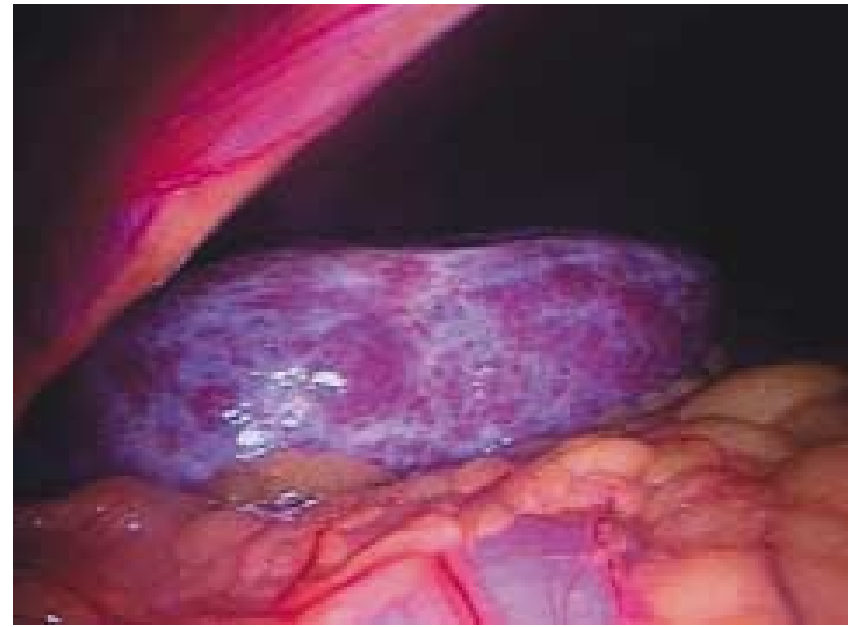

Figure 1 A 36-year-old Angolese man was examined because of portal hypertension of unknown origin. Physical examination revealed marked splenomegaly. Serological testing for schistosome infection was positive (enzyme-linked immunosorbent assay [ELISA] $1: 256)$. A laparoscopy was performed, and both right and left liver lobes showed a pattern of diffuse and irregular white bands, suggesting fibrosis.

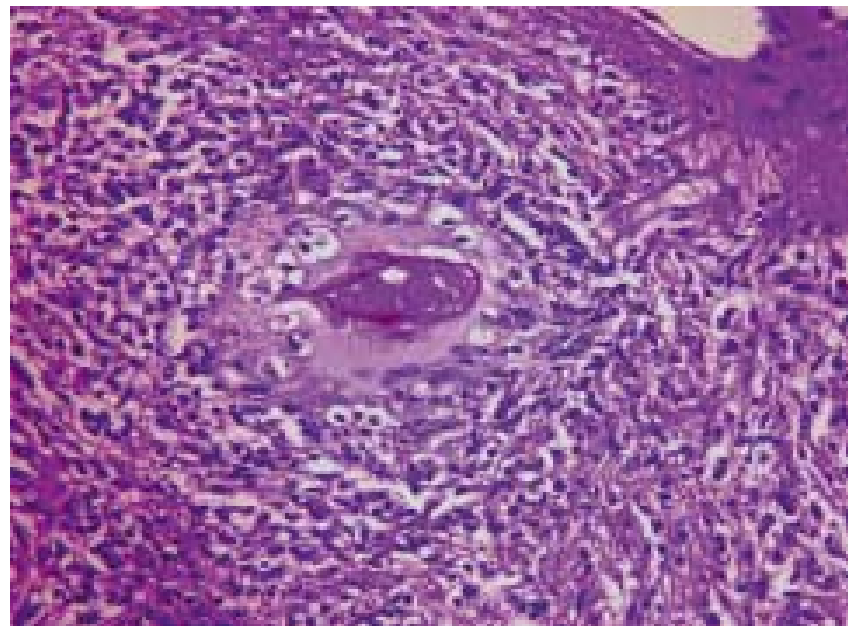

Figure 2 Microscopical examination of a liver biopsy showed periportal fibrosis and a schistosome egg was detected with a granulomatous reaction. Treatment with a single dose of praziquantel was given. 